\title{
Technology as a Source of Power: Exploring How ICT Use Contributes to the Social Inclusion of Refugees in Germany
}

\author{
Safa'a AbuJarour \\ University of Potsdam, Germany \\ safaa.abujarour@uni-potsdam.de
}

Hanna Krasnova

Weizenbaum Institute for the Networked Society and University of Potsdam, Germany krasnova@,uni-potsdam.de

\begin{abstract}
Since the beginning of the recent global refugee crisis, researchers have been tackling many of its associated aspects, investigating how we can help to alleviate this crisis, in particular, using ICTs capabilities. In our research, we investigated the use of ICT solutions by refugees to foster the social inclusion process in the host community. To tackle this topic, we conducted thirteen interviews with Syrian refugees in Germany. Our findings reveal different ICT usages by refugees and how these contribute to feeling empowered. Moreover, we show the sources of empowerment for refugees that are gained by ICT use. Finally, we identified the two types of social inclusion benefits that were derived from empowerment sources. Our results provide practical implications to different stakeholders and decision-makers on how ICT usage can empower refugees, which can foster the social inclusion of refugees, and what should be considered to support them in their integration effort.
\end{abstract}

\section{Introduction}

Over a million asylum seekers entered Europe in 2015, erupting a state of emergency all over Europe and sparkling the need to facilitate social inclusion of the newcomers. Referred to the state of "having the opportunities and resources to participate fully in economic, social and cultural life" [1], social inclusion represents a critical maxim of an equitable and democratic society.

From the very beginning, researchers have observed that this crisis is different from previous ones due to the significant reliance of refugees on technology, with the use of the Internet by refugees and asylum seekers reaching new records [2]. In response, IS studies have

\author{
Antonia Köster \\ Weizenbaum Institute for the Networked \\ Society and University of Potsdam, Germany \\ akoester@uni-potsdam.de \\ Manuel Wiesche \\ TU Dortmund, Germany \\ manuel.wiesche@tu-dortmund.de
}

focused on the potential of Information and Communication Technologies (ICTs) in alleviating the ongoing humanitarian crisis [3].

By investigating this new context, first research findings suggest that smartphones may have an empowering effect on refugees, serving as a primary gateway to keep the connection to others and access much-needed information to navigate the new reality [4]. Indeed, ICT-enabled services available on smartphones have continuously enabled refugees during the uncertain circumstances of their journey to Europe, in the first period of applying for asylum, and during the extended process of integration into the new society [5]. For example, [6] suggests that Internet use has helped refugees develop a much-needed sense of connectedness, thereby facilitating refugees' sense of agency and well-being. Additionally, ICT capabilities available to refugees have been linked to greater perceptions of emotional support, operational efficiency, and integration [5]. Overall, while initial evidence suggests that ICTs can contribute to the empowerment of refugees, thereby facilitating their social inclusion in the host country, the mechanism behind these effects is little understood. Specifically, it is not clear which features of ICT have an empowering influence on refugees and how this influence subsequently translates into social inclusion. To fill this research gap, in this study we build on the results of thirteen face-to-face interviews with Syrian refugees from Germany to investigate the mechanics of ICTenabled empowerment and its interaction with the refugees' perception of social inclusion.

We have chosen to focus on the population of Syrian refugees from Germany for two important reasons. First, Germany is the country of destination for the highest number of asylum seekers in Europe [7], and second, 
Syrian refugees represent the largest group of asylum seekers in Germany.

The rest of this paper is structured as follows. In section 2, we summarize the related work on the role of ICT use in the social inclusion of refugees and on empowerment components. In section 3 , we explain our methodology and describe the data collection and data analysis. In section 4 , we present our findings and the research model. In section 5, we discuss the results of our research. We conclude our study, highlight the limitations, and introduce recommendations for future research in section 6 .

\section{Related Work}

Recent studies suggest that modern ICTs can be the right tool to promote the social inclusion of refugees, enhance their well-being, and raise the personal sense of agency [5]. Nonetheless, the mechanisms behind these effects are still little understood (e.g., [4]). In this section, we summarize ongoing research into the role of ICTs in the social inclusion of refugees and introduce the concept of empowerment as a critical mechanism underlying this relationship.

\subsection{The Role of ICT Use in Social Inclusion of Refugees}

In the refugees' context, the goal of social inclusion is to grant opportunities for people to settle in, integrate, and participate in the new environment [5]. Recent research differentiates between two critical goals of social inclusion in the context of refugee integration: well-being and the sense of agency [8]. While wellbeing reflects individual "cognitive and affective evaluations of his or her life" [9, p. 187], agency is defined as the "freedom to set and pursue one's own goals and interests" [10]. Specifically, recent research stresses social networking, employment, education and language, health, government and citizenship, housing, and culture [5] as critical dimensions of agency in the refugee context.

Taking a closer look at the agency dimensions helps understand the intricacies of social inclusion processes in greater detail. Specifically, social networking underlies the sense of agency as it helps refugees develop a sense of belonging and get the emotional support they need [11]. Further, equal opportunities in the labor market and access to paid employment enable refugees to reach their economic self-sufficiency and regain a positive sense of identity and control over their lives $[12 ; 13]$. Equally, active participation in educational and language learning programs is vital to a successful integration process, with refugees often required to attend language courses upon arrival [14; 15]. Access to healthcare services is also viewed as essential, as refugees often lack information about their healthcare options, experience difficulty navigating the complex healthcare and insurance system, and are constrained in their communication abilities because of the limited language skills $[16 ; 17]$. In the same vein, the ability to navigate governmental offices and understand regulations as well as refugees' access to the housing market can further strengthen refugees' sense of agency on their path to integration. Indeed, refugees face numerous barriers when searching for a new home, including a lack of information about the housing market, limited awareness about the customs and strategies relevant to access housing, and insight into a complicated application process [13; 17]. Finally, participation in cultural activities enhances refugees' understanding of the host country's culture, thereby supporting their social inclusion [18; 19]. Generally, adapting to the new culture is highly correlated to refugees' overall satisfaction [20].

Notably, a growing body of research suggests that ICT solutions can support refugees in their integration journey and have the potential to tap into multiple domains of social inclusion [8]. Indeed, access to the Internet can provide refugees with general information about housing, employment, rights, citizenship, supporting services, language learning programs, and help overcome the feeling of isolation by supporting existing and establish new social connections $[5 ; 21$; 22]. For example, by facilitating relationships with others, smartphones can enable refugees to keep their connection to the family back home and help them initiate the first contact with the locals. Together, access to these old and new social networks can lessen refugees' perceptions of loneliness [23] and help them develop a sense of belonging to the new host country [6; $12 ; 24]$. Similarly, the use of translation applications helps refugees overcome existing language barriers, thereby opening new opportunities in their search for employment, healthcare options, and housing. For example, refugees rely on social media sites to understand and navigate the housing market and understand the local culture by providing useful tools and information [6]. Furthermore, refugees have been shown to actively use technology to connect with authorities to acquire information on regulations [8] as they apply and follow up on their asylum application [6; 25].

To sum up, with smartphone penetration rates among refugees reaching up to $90 \%$ [26], ICT can be seen as a fundamental means of empowering refugees on their path to integration [27]. 


\subsection{Empowerment}

Defined as "a process by which individuals gain mastery or control over their own lives and democratic participation in the life of their community" [28, p. 726], empowerment has been extensively discussed across a variety of different contexts and disciplines [29]. For instance, while the phenomenon of workplace empowerment has been in the focus of organizational studies (e.g. [30]), the concept of female empowerment has been studied in economics (e.g. [31]), and the idea of patient empowerment has gained significant recognition in the medical field [29].

Describing individual actions of those "with lesser power [to] gain control over their lives and influence the organizational and societal structures within which they live" [32, p. 215], empowerment is a multidimensional construct. Accordingly, researchers have described empowerment encompassing different dimensions (e.g., [32]), such as control, the achievement of goals, competence, critical awareness of the sociopolitical environment, self-esteem, and self-efficacy (e.g. [33]). While operationalizations differ depending on the research context and analysis level, three key dimensions of empowerment studied in the prior literature emerge as particularly salient: control, participation in the community, and self-efficacy. The control dimension refers to having control over one's life, future, and destiny, which leads to the feeling of being empowered [29]. The belief that one can control one's life events compared to the perception that one has no power to control important life outcomes is central to control [29]. The sense of control reflects the constraints and opportunities of one's position in society. For example, increasing responsibility and perceived control among nursing home residents resulted in happier, more active, and more alert residents [34].

Linked with a subjective sense of empowerment, participation in the community is described as being involved in organizations or communities [32]. Participation in communities, such as neighborhood groups, will help individuals develop skills by mimicking others and gaining knowledge through organizing, identifying resources, or developing strategies. [28; 33].

Finally, self-efficacy has been defined as the people's beliefs about their capabilities to attain specific types of performances and achieve particular results [35; 36]. As tasks vary in their level of difficulty, individuals often choose tasks based on their self-efficacy beliefs. Individuals with higher perceived self-efficacy will set more difficult challenges for themselves [36]. Thus, self-efficacy thinking has been linked to motivation processes and performance [36].
Importantly, empowerment has been associated with several positive outcomes, some of which can be situated in the domain of social inclusion. For example, empowerment has been found to be related to life satisfaction [37] and quality of life [29]. Additionally, researchers have linked a sense of personal control to a greater level of health and well-being [38]. Involvement in community organizations and activities has been found to be linked to feeling less alienated [39]. Additionally, self-efficacy has been shown to significantly impact the enhancement of human accomplishment and personal well-being [36]. At the same time, people with a low sense of self-efficacy also tend to have low self-esteem that may lead to depression [36].

Looking into the role of ICTs, extant IS literature has so far focused on uncovering the means of crowd worker empowerment (e.g. [40]) and how social media empowers communities during crisis response [41]. However, the empowering potential of ICT in the context of social inclusion has received little attention. For example, it has been indicated that a lack of power can leave refugees in the lowest-paid jobs, working long hours, often isolated, and without any opportunity to contest their situation, which leads them to be socially excluded from the host community [42]. Moreover, refugees are often not able to control their destinies as they have been forcibly displaced [43]. Therefore, it is essential to unravel the means of accentuating refugee empowerment through ICTs.

Building on these research results, and due to the high reliance of refugees on services enabled by ICT solutions, in this study, we set out to explore whether and how ICT use by refugees may contribute to their sense of empowerment, and thereby their perception of social inclusion in the host society.

\section{Methodology and Sampling}

\subsection{Methodology}

As the phenomenon being studied has not received significant theoretical research attention, we chose a qualitative research methodology to obtain initial insights into understanding how ICT usage leads to empowerment formation, and how feeling empowered leads subsequently to social inclusion. Qualitative research is particularly suitable to explore a phenomenon in its natural context [44]. In our study, we adopt the coding-strategies from Grounded Theory Methodology [44]. These procedures helped us analyze the available data systematically and uncover the underlying relationships in the data. 


\subsection{Data Collection and Sampling}

To gain an in-depth understanding of refugees' ICT usage, thirteen face-to-face interviews with Syrian refugees were carried out in the area of Berlin, Germany. All interviewees were recruited and conducted personally by the first author, who has direct access to refugee networks and communities. Eight interviewees were male, and five were female. Most of our interviewees have arrived in Germany at least 2-3 years prior to the interviews, and only two had been in Germany for less than a year at the time of the interview. Almost every respondent already had a decision on the residence permit, while only one respondent had not yet received a decision. All refugees still had family members in Syria at the time of our study.

The average duration of the interviews was 49 minutes. All interviews were initially conducted in Arabic (the native language of the interviewees and the first author) and audio-recorded. In the next step, they were transcribed into a text document and then carefully translated into English. Two authors of the study did the coding to ensure consistency of the emerging concepts and categories.

The interviews were conducted following a semistructured approach. We asked respondents questions related to their journey to Europe, their current situation in Germany, how they have been using different types of ICTs during their journey and after their arrival, their participation in educational programs, how they use Social Media, and their perceptions of social inclusion in their new home. Further questions were related to their feelings when they are able or unable to access or use their smartphone. We asked interviewees openended questions that allowed them to provide their perspectives on the dynamics of ICT usage and empowerment. In all interviews, follow-up questions were asked to ascertain the appropriate context and attain a better understanding.

\subsection{Data Analysis}

We followed the 'Straussian' line of Grounded Theory [44] for the coding process, which allows for incorporating prior knowledge of the phenomenon in question into the analysis $[44 ; 45 ; 46]$. In their approach, Strauss and Corbin [44] differentiate between three major types of coding: open coding, axial coding, and selective coding.

We started with open coding, which pertains specifically to the naming and categorizing of phenomena through line-by-line coding of all data. This involves close examination, phrase by phrase, and even sometimes of single words. Initial concepts were identified by looking for patterns in the data through the process of constant comparison [44]. In the next step, the concepts identified during open coding were grouped under higher-order, more abstract concepts, called categories.

The concepts identified were combined to higherlevel categories that relate to ICT tools and usages, empowerment dimensions, and social inclusion benefits. We further linked the major categories to develop a coherent perspective on our data. Following the constant comparison principle [47], we returned to the data whenever a relationship emerged in the selective coding to verify its grounding in the data.

\section{Findings and Research Model}

In this section, we analyze our qualitative data and show quotes $(\mathrm{Q})$ from our interviews with refugees.

\subsection{ICT Use}

In the current refugee crisis, the high reliance of refugees on the ICT is expected to provide enormous benefits for refugees. Our interviews showed that ICT, especially the smartphone, is an essential part of refugees' lives in the host country: "[...] Without the smartphone it would have been a big disaster" (Q). Another respondent describes the feeling of the increased value of the smartphone: "My smartphone has a big value and I cannot live without it" $(\mathrm{Q})$.

Our interviewees revealed several applications that they are using on smartphones, varying from communication applications, language learning and translation, accessing information and expanding knowledge, etc. Some examples include: "[...] I use Facebook, Viber, WhatsApp, and other applications. I communicate with friends, and I like to follow the news" (Q). "I use YouTube a lot. You can find everything you are looking for on YouTube" (Q).

Moreover, it was clear from the interviews that refugees feel empowered by using ICT solutions, especially smartphones and having an Internet connection. One respondent revealed: "The smartphone empowers me when I need to know something and it facilitates this" (Q). Refugees are also aware of the risks of smartphone addiction. However, the empowerment they receive has a higher value: "I do not feel that my smartphone controls me. On the contrary, it empowers me" $(\mathrm{Q})$, or "There are two ways: the smartphone controls me, or I control it. There are a few important things to me [in life]; to learn the language and to learn about the rules in this country. Here comes the importance of the smartphone. Hence, it can be said that it depends on the way of using the smartphone" (Q). One respondent summarized the value of the smartphone and its functions: "I need the smartphone to 
learn the language, know the roads, and communicate with people here and in Syria. If I were in my home country, I would be among my parents, relatives, and friends, and I would use the smartphone for normal calls only. Besides, I wouldn't need translation applications and to use Facebook a lot. Here in Germany, on the contrary, it is essential" (Q).

Our interviews showed that refugees use ICT tools for communication purposes. Firstly, it enables communicating with family and friends in the home country: "I use it [the smartphone] to communicate with my family via Facebook, WhatsApp, and sometimes I use Viber. I also use it to communicate with my friends" (Q). Another interviewee noted: "The smartphone is an essential part for me here (in Germany) as a refugee because I have to communicate with my family (back home). For example, in the past, I used to open WhatsApp once a week, because I was communicating with my friends on the ground and my family were close to me, so I didn't need it. As for me here, the only way to communicate is the smartphone" $(\mathrm{Q})$. Secondly, it enables communicating with the local community in the host country: "I used an app called 'Berlin Dinner' on my smartphone. My teacher at school advised me to use it. Through this app, I met a German girl" (Q) and "I also use the smartphone to communicate with my German friends" (Q). Using ICT solutions for communication with family and friends back home as well as with the local community in the host country contributes to the sense of empowerment for refugees and supports their social inclusion efforts.

\subsection{Empowerment Dimensions}

4.2.1. Community Participation. Participation in the community, expressed in refugees' willingness to be part of a society and give back to the community, emerged as an important source of empowerment. Our interviews showed that ICTs enable refugees to participate in the community in two ways: first, by connecting them to their friends and family back home, and second, by connecting them to the local community (to Germans in our current study). To reflect refugees' participation in these two communities, we refer to this construct as "Community Participation" in our model.

Our interviews showed the importance of refugees' social connectedness with family and friends back home, as it makes them feel connected and cared about [48] and can have a positive impact on their social and health outcomes. For instance, one refugee responded: "Of course, communicating with my family affects my learning, and it affects everything. For example, [without the communication] if I have an appointment, I don't feel like attending it, or if I have to achieve some work, I don't feel able to get it done. I would not be able to do anything unless I communicate with my family" (Q). Moreover, not being able to communicate has a negative influence on the well-being of refugees in our sample "The smartphone is very valuable for me. It's something important in our daily life here and for the members of our family to communicate with each other, and without it, I feel lost and lonely" (Q).

Importantly, being connected to the local community of Germans was also very relevant for our interviewees. Indeed, previous research shows that being socially connected to a network of locals helps refugees feel less stressed in the integration process, enhances their knowledge about the new host society, its social norms and cultural practices [13], as well as promotes one's identity and a sense of belonging [49]. Our interviews confirm that ICT usage facilitates community participation with Germans: "For instance, I got to know several German friends through the Internet, and we have been in touch, although we did not know each other before" $(\mathrm{Q})$. As a result, this ability to be connected had an empowering impact: "In general, one of the sources of power for me is being able to be in touch with Germans" (Q). Further, another interviewee noted: "In general, among the things that empower me are communicating with the society, having a desire to work and have a job, but not feeling that I'm a burden on society. I need to be useful to society and participate in its construction because I love to work and consider it a source of strength for me" (Q). Through networking with others in the local community, refugees can also obtain access to resources contained in these networks. These resources usually relate to getting information or asking for favors, such as accommodation during travel, putting in contact with someone, or helping with finding employment. Thus, our interviewees felt that they could obtain networking benefits by using the smartphone: "I can say that the smartphone is the means of communication between me and the people that I get to know, who empower me" (Q).

Additionally, being connected to Germans enabled learning about the values and culture of the host country "[power] is being in touch with Germans to learn about the traditions and customs of the community I am living in. This affects me positively and empowers me because I would love to be an active member in the society and integrate into it to get rid of the feelings of isolation and of being a stranger" (Q).

Our findings show that participation in the community is a vital component of refugee empowerment. On the one hand, refugees need to stay connected to the network of family and friends back home. On the other hand, refugees are being connected to the local community in the host country. Community participation through ICTs contributes to the social inclusion process of refugees in that it facilitates 
agency-related factors and positively affects their wellbeing, as we show in section 4.3.

4.2.2. Control. Following our interviews, the ability to use the smartphone functionalities anytime and anywhere gives refugees an empowering feeling of control through connectivity: "Of course, the smartphone can give me a sense of power and control over my life" (Q). This feeling is very critical for refugees as they cope with uncertain and continually changing circumstances. Another respondent described the power of the smartphone as follows: "It [the smartphone] empowers me. Anything that I want to do, the smartphone provides" (Q).

Moreover, having access to information gives refugees a greater sense of control. Moreover, access to necessary information is also an essential component for achieving social inclusion [24]. Indeed, refugees need information on various topics, and being informed gives them orientation and a sense of control: "The smartphone gives me a sense of control and power over the circumstances surrounding me. For example, if I want to go to an area, and I do not know how to do it, I simply put the address on Google Maps, and it directs $m e "(\mathrm{Q})$.

Our interviews show that having the smartphone always available and depending on it when needed gives the feeling of control and safety: "Well, it [the smartphone] is everything for me. I depend on it to a large extent all the time. Without it, I would get lost. I use it when I move from one place to another. When I use my smartphone for transportation, I feel more comfortable, relaxed, and relieved compared to taking a taxi, where I do not feel that safe" (Q).

In general, our interviews showed that the functionalities that the smartphone provides are a great source of control over the situation of life, which then contributes to the feeling of empowerment for the refugees: "Of course, the smartphone provides me with the feeling of control over a lot of situations. For example, by accessing the bank account, providing information about searching for accommodation, and even information about turning the smart TV on and off, also communicating with my family back home. I actually control a lot of things through the smartphone" (Q).

4.2.3. Self-Efficacy. A strong sense of efficacy enhances human accomplishment and personal wellbeing because people with high assurance in their capabilities approach difficult tasks as challenges to be mastered rather than as threats to be avoided [36]. Our interviews showed that using ICT contributes positively to the sense of self-efficacy among refugees: "The smartphone gives me a sense of power because through it I can get/do whatever I want" (Q). Moreover, one interviewee mentioned that the capabilities provided through the smartphone became even more critical after arriving in Germany, indicating the empowering value of smartphones to refugees: "The smartphone was important to me since I was in Syria and became more important after my arrival to Germany. I use it to communicate with others, for translation services, and to navigate through the city. Without the smartphone, it would have been a big disaster" (Q). Our interviews showed that refugees had developed a sense of selfefficacy by using their smartphones to cope with various situations: "Having my smartphone available for me at any moment I need enables me to do whatever I want from wherever I'm" (Q).

Moreover, the ability to use the smartphone for different purposes might enhance the personal capabilities of refugees [8]. One refugee explained: "Because using [the smartphone], I learn new things, expand my knowledge and interact more effectively with my surrounding. I'm sure if I'm still in Syria, I wouldn't have a smartphone, and my house would have been not connected to the Internet" $(\mathrm{Q})$. Thus, smartphone usage might also broaden the horizon of refugees, that master their lives in a new country with the help of ICTs.

Another very important capability achieved through the smartphone is learning. For instance, language skills are critical for attaining independent social functioning and social inclusion: "In general, things that give me power or are the source of power for me are learning the German language and getting the certificate of BI [level] or even the certificate of B2 [level]. Such that I would be able to work. It is important for me to feel strong, being able to work and earn money as I used to do in Syria. Thus, I can do whatever I want without being controlled by anyone else" (Q).

Taken together, our interviews provide evidence for the ability of ICTs, especially smartphones with their diverse functionalities, access to the Internet and Social Media, to empower refugees with a much-needed sense of control, self-efficacy, and the ability to reach the communities both back home and in a host country. Together, these sources of refugee empowerment provide a fertile ground for the benefits of social inclusion, as explained in the following section.

\subsection{Social Inclusion Benefits}

4.3.1. Agency. Our interviews showed that the absence of the family had a negative effect on refugees, which leads to being unable to fully engage in the social inclusion process. The smartphone has helped to smooth the situation, but feeling empowered to be able to build a new life in the host country was still missing. One respondent explained: "[When] my family was not with 
me, where I was only able to see the pictures of my kids through my smartphone, knowing they were in a dangerous place, [...] I knew that it could happen any time that I won't be able to communicate with them. All of this had a big negative impact on me. I didn't want to do anything, and I wasn't able to. I wasn't able to learn like others who have their families here with them, and they go to the school or even when they learn via elearning channels on YouTube. For me, I had the will to learn, but I couldn't."

On the other hand, our interviews revealed an interesting finding that when refugees feel empowered, also through using the smartphone to communicate, it consequently leads to achieving agency-related aspects that lead to social inclusion. One respondent explained: "Communicating with my family [via the smartphone] makes me more powerful. My mother always supports me and empowers me to be able to learn the language and work" (Q). This emphasizes the important role that the feeling of empowerment plays in the social inclusion of refugees in the host country.

Feeling empowered facilitate achieving social inclusion benefits. For instance, the sense of power through communicating with Germans lead to agencyrelated aspects of social inclusion: "I communicate with a German woman, who helped us finding a house, enrolling my daughter in kindergarten, and finding spots in the language schools" (Q).

We find that the smartphone enables refugees to expand their knowledge to enhance their sense of agency: "It is also possible to get to know the regulations of the country (Germany) in Arabic through the smartphone, as there are explanations about this topic provided online by Arab teachers" (Q).

In the refugee context, research has shown that active participation in education is essential to their successful integration [18], which also contributes to their sense of agency. Another respondent expressed the capability achieved by the smartphone in learning the language: "In general, one of the things that empower me is mastering the language. Without mastering the language, one can do nothing here. Although my language level is not that good, during the last five months, I felt a totally different person. I can understand what is going around me and communicate with people. I do not understand all the words, but I can understand the context. Before that, I was so weak, and I experienced several situations where I did not understand what was happening and how things happen in this country" (Q). Moreover, one refugee stated that the smartphone facilitates knowledge building anytime: "During language classes, [...] if I do not understand a particular rule, I would be able to look it up on YouTube and understand it, when I get home. Without a smartphone, it would be very difficult to do that" (Q).
After gaining some level of the host language skills, refugees also use their smartphones to analyze the labor market and to search for paid employment, which improves their sense of agency, and consequently, their social inclusion: "It would be possible after getting B1 [level] certificate to look for training, to look for a job and to know what the requirements are to have the job I want. All of that can be done through the smartphone" (Q).

4.3.2. Well-Being. Our interviews showed that the smartphone contributes to the community building with family, which is part of refugees' empowerment while losing this empowerment affects refugees' well-being: "Losing my smartphone would be very difficult for me. In particular, as I live in a new country and I can contact my family through the smartphone, only. So, that would have a negative psychological impact on me" (Q).

Additionally, our interviews showed that using ICT as an empowerment tool that contributes to the social inclusion of refugees leads to a higher quality of life for refugees. This is happening on different levels. For instance, refugees perceive access to information as a necessity in their new lives: "I would feel lost and depressed if I would lose my smartphone. I can't live alone and isolated from society. It is a necessity, and it provides me with whatever information I search for" (Q). Another refugee explained that the smartphone helps them to manage their lives: "It [smartphone] is very necessary; it has a main role in life management" (Q). Furthermore, one refugee even described that the smartphone does not only provide value to him through its inherent functions but also spills over to his functioning in life: "The smartphone is highly valuable for me. Without it, I wouldn't be able to focus because it is highly connected to my life" (Q) or as another refugee described: "I feel lost without a smartphone and Internet, especially while living in a developed country like Germany”(Q).

Moreover, our qualitative study also showed that the positive feeling is continuing, and refugees even feel that social connectedness via their smartphones enhances their life satisfaction and gives them a positive outlook for the future: "Communicating with my family through the smartphone affects my positive outlook for the future. As long as there is communication (with my family), positivity prevails-there is no positive life without being able to communicate with them" (Q). Our interviewees revealed some differences in their current ICT usage in the host country to their usage in their home countries, which contributes to their satisfaction with the current lives: "Smartphones became an essential part of our daily life. [...] In Arab countries, there is a rich social life that leaves no space for spending a lot of time using the mobile. In Germany, 
there is much free time that can be invested in learning the language [for example]. The smartphone will facilitate that through translation applications and other applications to know how to move from one place to another. They are also essential for communicating with parents in Syria" (Q).

Additionally, the support granted by being connected contains the emotional comfort of other people in one's network. Engaging with one's family can contribute to engagement in the local community and broader society because they provide the needed emotional support to refugees, which build new lives in the host country $[12 ; 24]$. Communication with the family back home has a positive effect on well-being, as it reduces depression and the feeling of isolation, which can occur when refugees cannot contact their family via the smartphone: "When I try to call my family, and no one answers, the first thing comes to my mind is that something bad happened and I feel depressed. Also, when I speak with my mother, I feel so happy" (Q).

These findings show that having a smartphone can enrich the life of refugees beyond the value of pure smartphone functionalities. It shows how important support is to motivate refugees to achieve well-being as well as agency-related aspects of social inclusion.

Based on extensive data analysis, we formulate a research model depicted in Figure 1, which differentiates between ICT usage as a causal condition of empowerment dimensions that lead to achieving social inclusion benefits.

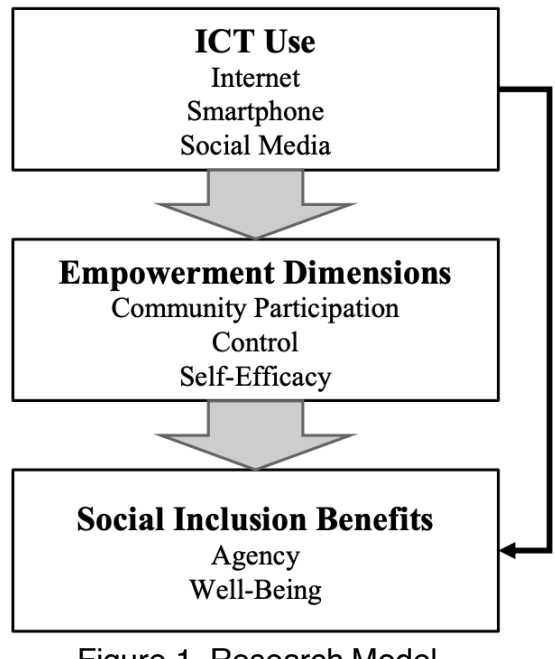

Figure 1. Research Model

\section{Discussion}

Results from our qualitative study revealed that the ability to use the smartphone functionalities anytime and anywhere gives refugees an empowering feeling of global connectivity, which is critical for refugees as they cope with uncertain and continually changing circumstances. Literature shows that empowerment is highly related to well-being and life satisfaction, where empowerment has significant positive correlations to quality of life [29] and where greater perceived empowerment leads to higher overall individual life satisfaction [37]. Our results identified the need for refugees to be connected with the family and friends back home and with the local community in Germany because being connected to these groups has a positive impact on the refugee lives, and not being connected to them negatively affects their well-being. Additionally, our results showed that being connected to the family contributes to the refugees' positive outlook and gives the power to cope with the new situations, and having contacts with German friends positively affects the sense of belongings and, thus, the overall process of social inclusion.

Based on a literature review, we identified seven common dimensions of social inclusion of refugees in terms of agency [6]: social networking, employment, education and language, culture, health, government and citizenship, and housing. Our interviews revealed another dimension to the existing body of knowledge on refugee agency, namely, transportation. This new dimension is important for refugees that describe their ability to use transportation services that can be enabled by the smartphone: "When I use my smartphone for transportation, I feel more comfortable, relaxed and relieved" $(\mathrm{Q})$.

Another important aspect is having access to information through ICT, which ensures that refugees can participate in two communities simultaneously. Having access to the needed information on various topics and being informed gives refugees orientation and a sense of control, which are essential for empowering refugees, and consequently, contributing to the social inclusion process. By accessing this source of empowerment, refugees can feel in control and that they have mastery over their lives and decisions. This leads to achieving the desired benefits of social inclusion, such as finding suitable job opportunities, participating in language learning and educational programs, navigating through the bureaucratic system, and coping with the new culture and way of life.

Our findings showed that having a smartphone can enrich refugees' lives beyond the value of pure smartphone functionalities and contribute positively to their higher quality of life and life satisfaction. Our empirical study contributes to the body of knowledge on empowerment by showing how ICTs are driving empowerment dimensions by facilitating two directions of community participation that are specific to the context of refugees, by enabling a higher level of control over one's life, and by positively contributing to one's self-efficacy. 
We believe that these findings are important for different stakeholders related to the refugee topic. These include (a) governments; to understand how refugees use the smartphone and try to provide targeted services that can help refugees to navigate through the official systems, and (b) industry; to work on more user-centric ICT solutions that target this specific group of users and offer solutions based on their needs and requirements.

\section{Conclusion, Limitations, and Next Steps}

In our study, we investigated the role of ICT use, particularly the smartphone, in the social inclusion of Syrian refugees in Germany and its contribution to their feeling of power. In this paper, we identified two types of social inclusion benefits that refugees can gain from ICT use in terms of agency and well-being. Furthermore, we showed ICT contributes to empowering refugees on three levels; community participation, sense of control, and self-efficacy, which are the most crucial empowerment components and drivers that subscribe to refugees' social inclusion in the new society.

Our research is limited in the sample size. Although the analysis of our interviews showed coherency in the sample, further investigation is needed to verify whether the results are generalizable to the entire population of Syrian refugees in Germany. Moreover, since we included only Syrian refugees in our scope, our results did not cover the refugee population from different countries of origin. Considering the urgent need to understand ICT use in the context of refugees, the authors plan to investigate further how ICT-enabled services can contribute to the overall feeling of empowerment with a larger sample.

The next steps of our research include conducting more interviews to gain more in-depth insights into the topic with the purpose to (1) dive deeper into the usage of ICT tools by refugees, especially smartphone apps, (2) find further links between ICT and empowerment, and (3) define the most relevant social inclusion benefits that are derived from feeling empowered through ICT use by refugees. Moreover, in our future research, we will consider community context, culture, and crosscultural communication barriers as well as facilitators of the ICT usage by refugees.

\section{Acknowledgment}

Part of this work has been funded by the Federal Ministry of Education and Research of Germany (BMBF) under grant no. 16DII116 ("Deutsches Internet-Institut").

\section{References}

[1] C. Wilson, and J. Secker, "Validation of the Social Inclusion Scale with Students," Social Inclusion. (3:4), pp. 52-62. 2015.

[2] D. WELT, "Datenverbrauch von Flüchtlingen übersteigt den von Airports", Retrieved from http:/www.welt.de/wirtschaft/article152422950/Datenv erbrauch-von-Fluechtlingen-uebersteigt-den-vonAirports.html, 2016.

[3] S. AbuJarour, M. Wiesche, A. Díaz Andrade, J. Fedorowicz, H. Krasnova, S. Olbrich, C.-W. Tan, C. Urquhart, V. Venkatesh, "ICT-Enabled Refugee Integration: A Research Agenda," Communications of the Association for Information Systems. Vol. 44, pp. 874-891, 2019.

[4] A. C. Egan, "The psychological impact of smartphones: The effect of access to one's smartphone on psychological power, risk taking, cheating, and moral orientation," Ph.D. thesis, Loyola University Chicago, 2016.

[5] S. AbuJarour, and H. Krasnova, "Understanding the role of ICTs in promoting social inclusion: The case of Syrian refugees in Germany," in European Conference on Information Systems (ECIS), Guimarães, Portugal, 2017.

[6] S. AbuJarour, H. Krasnova, and F. Hoffmeier, "ICT as an Enabler: Understanding the Role of Online Communication in the Social Inclusion of Syrian Refugees in Germany," European Conference on Information Systems, Portsmouth, UK, 2018.

[7] UNHCR, "Persons of concern," Retrieved from http://popstats.unhcr.org/en/persons_of_concern, 2020.

[8] A. Diaz Andrade and B. Doolin, "Information and communication technology and the social inclusion of refugees," MIS Quarterly, vol. 40, no. 2, pp. 405-416, 2016.

[9] E. Diener, D. Kahneman, R. Arora, J. Harter, and W. Tov, "Income's Differential Influence on Judgments of Life Versus AffectiveWell-Being," in Assessing Well-Being, E. Diener (ed.), New York: Springer, pp. 233-246, 2009.

[10] A. Sen, "Well-Being, Agency and Freedom: The Dewey Lectures 1984," The Journal of Philosophy, vol. 82, no. 4, pp. 169-221, 1985.

[11] H. Beirens, N. Hughes, R. Hek, and N. Spicer, "Preventing Social Exclusion of Refugee and Asylum Seeking Children: Building New Networks," Social Policy and Society. (6:02), 2007.

[12] M. Beiser, A. M. Goodwill, P. Albanese, K. McShane, and P. Kanthasamy, "Predictors of the Integration of Sri Lankan Tamil Refugees in Canada: Pre-Migration Adversity, Mental Health, Personal Attributes, and PostMigration Experience," International Journal of Migration, Health, and Social Care, 2015.

[13] F. Fozdar and L. Hartley, "Refugee resettlement in Australia: What we know and need to know," Refugee Survey Quarterly, vol. 32, no. 3, pp. 23-51, 2013.

[14] A. Ager and A. Strang, "Understanding Integration: A Conceptual Framework," Journal of Refugee Studies, vol. 21, pp. 166-191, 042008.

[15] S. Yu, E. Ouellet, and A. Warmington, "Refugee Integration in Canada: A Survey of Empirical Evidence 
and Existing Services," Refuge: Canada's Journal on Refugees, vol. 24, pp. 17-34, 2007.

[16] V. Colic-Peisker, "'At least you're the right colour': Identity and social inclusion of Bosnian refugees in Australia," Journal of Ethnic and Migration Studies 31.4, 615-638, 2005.

[17] E. Mestheneos, and E. Ioannidi, "Obstacles to Refugee Integration in the European Union Member States," Journal of Refugee Studies. (15:3), pp. 304-320, 2002.

[18] N. Ives, "More Than a "Good Back": Looking for Integration in Refugee Resettlement" Refuge: Canada's Journal on Refugees, pp. 54-63, 2007.

[19] M. Stewart, L. Simich, M. Beiser, K. Makumbe, E. Makwarimba, and E. Shizha, "Impacts of a Social Support Intervention for Somali and Sudanese Refugees in Canada," Ethnicity and Inequalities in Health and Social Care. (4:4), pp. 186-199, 2011.

[20] V. Colic-Peisker, "Visibility, settlement success and life satisfaction in three refugee communities in Australia," Ethnicities 9, no. 2: 175-199, 2009.

[21] A. Alencar, "Refugee integration and social media: A local and experiential perspective," Information, Communication \& Society, vol. 21, no. 11, 2018.

[22] E. McGregor, M. Siegel, et al., "Social media and migration research," tech. rep., United Nations University- Maastricht Economic and Social Research Institute, 2013.

[23] L. Komito, "Social media and migration: Virtual community 2.0," Journal of the American Society for Information Science \& Technology, vol. 62, no. 6, 2011.

[24] J. Aumüller and C. Bretl, "Die Kommunale Integration von Flüchtlingen in Deutschland." Berliner Institut für Vergleichende Sozialforschung - Berlin, 2008.

[25] Schreieck, M., Zitzelsberger, J., Siepe, S., Wiesche, M., and Krcmar, H. "Supporting Refugees in Everyday LifeIntercultural Design Evaluation of an Application for Local Information," PACIS Proceedings, 2017.

[26] C. Maitland and Y. Xu, "A social informatics analysis of refugee mobile phone use: A case study of Za'atari Syrian refugee camp," TPRC, 2015.

[27] M. Brunwasser, "Migrant essentials extend to smartphone," International New York Times. Retrieved from www.nytimes.com/2015/08/26/world/europe/a21 st-century-migrants-checklist-water-sheltersmartphone.html, 2015.

[28] S. P. Segal, C. Silverman, and T. Temkin, "Measuring empowerment in client-run self-help agencies," Community Mental Health Journal, vol. 31, no. 3, pp. 215-227, 1995.

[29] E.S. Rogers, J. Chamberlin, M.L. Ellison, and T. Crean, "A consumer-constructed scale to measure empowerment among users of mental health services," Psychiatric Services, vol. 48, no. 8, pp. 1042-1047, 1997.

[30] G. M. Spreitzer, "Psychological empowerment in the workplace: Dimensions, measurement, and validation," Academy of Management Journal, vol. 38, no. 5, 1995.

[31] E. Duflo, "Women empowerment and economic development," Journal of Economic Literature, vol. 50, no. 4, pp. 1051-79, 2012.
[32] S. P. Segal, C. Silverman, and T. Temkin, "Measuring empowerment in client-run self-help agencies," Community Mental Health Journal, vol. 31, no. 3, pp. 215-227, 1995.

[33] M.A. Zimmerman, "Empowerment Theory," In Rappaport J., Seidman E. (eds.) Handbook of Community Psychology. Springer, Boston, MA, 2000.

[34] E. J., Langer, and J. Rodin, "The effects of choice and enhanced personal responsibility for the aged: A field experiment in an institutional setting," Journal of Personality \& Social Psychology, 34(2), 191-198, 1976.

[35] I. Ajzen, "Perceived behavioral control, self-efficacy, locus of control, and the theory of planned behavior 1," Journal of applied social psychology, vol. 32, no. 4, 2002.

[36] A. Bandura, "Self-efficacy," Encyclopedia of Human Behavior, pp. 1-3, 1994.

[37] S. Rosenfield, "Factors contributing to the subjective quality of life of the chronic mentally ill," Journal of Health and Social Behavior, pp. 299-315, 1992.

[38] J. M. Griffin, R. Fuhrer, S. A. Stansfeld, \& M. Marmot, "The importance of low control at work and home on depression and anxiety: Do these effects vary by gender and social class?" Journal of Social Science \& Medicine, 54(5), 783-798, 2002.

[39] H. Levens, "Organizational affdiation and powerlessness: A case study of the welfare poor," Social Problems, 16, 1968.

[40] X. N. Deng, and K. D. Joshi, "Is crowdsourcing a source of worker empowerment or exploitation? understanding crowd workers' perceptions of crowdsourcing career", 2013.

[41] C.M.L. Leong, S. L. Pan, P. Ractham, and L, Kaewkitipong, "ICT-Enabled Community Empowerment in Crisis Response: Social Media in Thailand Flooding 2011" Journal of the Association for Information Systems: Vol. 16: Iss. 3, Article 1, 2015.

[42] A. Bloch, "The labour market experiences and strategies of young undocumented migrants," Work, Employment and Society, vol. 27, no. 2, pp. 272-287, 2013.

[43] L. Malkki, "Speechless Emissaries: Refugees," Humanitarianism, and Dehistoricization. Cultural Anthropology, 11(3), 377-404, 1996

[44] A. Strauss and J. Corbin, "Basics of qualitative research: Grounded theory," Procedures and Techniques, 1990.

[45] R. Matavire and I. Brown, "Profiling grounded theory approaches in information systems research," European Journal of Information Systems, vol. 22, no. 1, 2013.

[46] S. Seidel and J. C. Recker, "Using grounded theory for studying business process management phenomena," Association for Information Systems, 2009.

[47] C. Urquhart, H. Lehmann, and M. D. Myers, "Putting the 'theory' back into grounded theory: guidelines for grounded theory studies in information systems," Information systems journal, vol. 20, no. 4, 2010.

[48] A. S. I. Board, Social inclusion in Australia: How Australia is faring. Department of the Prime Minister and Cabinet, 2012.

[49] P. Flanagan, S. Garry, and J. A. Jason, "Housing and inclusion." A Life in the Day, vol. 10, issue 1, 2006. 